\title{
ESTIMATING THE SENSITIVITY OF THE ALGEBRAIC STRUCTURE OF PENCILS WITH SIMPLE EIGENVALUE ESTIMATES*
}

\author{
DANIEL BOLEY ${ }^{\dagger}$
}

\begin{abstract}
The sensitivity of the algebraic (Kronecker) structure of rectangular matrix pencils to perturbations in the coefficients is examined. Eigenvalue perturbation bounds in the spirit of Bauer-Fike are used to develop computational upper and lower bounds on the distance from a given pencil to one with a qualitatively different Kronecker structure.
\end{abstract}

Key words. matrix pencil, controllability, sensitivity, distance to uncontrollability, linear dynamical systems, Kronecker canonical form

AMS (MOS) subject classifications. 15A22, 15A21, 93B05, 93B40, 65F30

1. Introduction. In this paper, the sensitivity of the algebraic (Kronecker) structure of rectangular matrix pencils to perturbations in the coefficients is examined. Eigenvalue perturbation bounds in the spirit of Bauer-Fike are used to develop computational upper and lower bounds on the distance from a given pencil to one with a qualitatively different Kronecker structure. A note on notation: All norms $\|\cdot\|$ used in this paper are the vector or matrix 2-norm, as appropriate.

The main goal of this paper is to present some results regarding matrix pencils, of the form $A-\lambda B$, where $\lambda$ is a free parameter and $A, B$ are $n \times p$ matrices with $n>p$. In the classical theory of matrix pencils [8], [11], it is well known that any pencil is equivalent to its Kronecker Canonical Form (KCF), which is a pseudodiagonal matrix with diagonal blocks of the form $L, L^{T}$, and/or $J$, where

$$
L=\left[\begin{array}{c}
I \\
{[0, \cdots, 0]}
\end{array}\right]+\lambda\left[\begin{array}{c}
{[0, \cdots, 0]} \\
I
\end{array}\right]
$$

is a matrix with one more row than column, and $J$ is a square matrix in Jordan Canonical Form. We call $L$ a "tall-thin" K-block, $L^{T}$ a "short-fat" K-block, and $J$ the "regular" part.

In this paper, we deal exclusively with tall-thin pencils. Such pencils always have at least $n-p$ tall-thin K-blocks. In [3], we showed that the set of all tall-thin pencils with only tall-thin K-blocks is open and dense in the set of all pencils of the same shape. Hence, given a tall-thin pencil, the question we attempt to address is if it has any other types of K-blocks, and if not, what is the distance to the nearest pencil which does. In [15] and [10], algorithms were proposed that compute the complete $\mathrm{KCF}$ for a given pencil guaranteed to be exact for a pencil close to the original given pencil (backward stable). If the KCF computed in this way has only tall-thin K-blocks (the "generic case"), then one is still left with determining how far it is from a pencil with other types of K-blocks. In this paper, we attempt to estimate this distance from both above and below. A detailed algebraic analysis for square pencils was given by Waterhouse [17], but beyond that surprisingly little has been found in the literature on this topic.

\footnotetext{
* Received by the editors June 27, 1988; accepted for publication (in final revised form) November 2, 1989.

$\dagger$ Computer Science Department, University of Minnesota, Minneapolis, Minnesota 55455. (boley@umn-cs.cs.umn.edu). This research was partially supported by National Science Foundation grants DCR-8420935, CCR-8813493, and DCR-8519029.
} 
We use the following characterization of the Kronecker structure of a pencil.

Definition 1. A matrix pencil $A-\lambda B$ is said to be deficient if there exists some $\lambda$ for which it is not full rank, where $\lambda$ is a complex number or "infinity." If $A-\lambda B$ is always full rank for any value of $\lambda$, then it is said to be nondeficient.

All tall-thin pencils have at least one tall-thin $(L)$ K-block. The condition that the pencil be deficient is equivalent to the existence of at least one value $\lambda$ and vector $\mathbf{x}$ such that $(A-\lambda B) \mathbf{x}=0$, and it corresponds to the existence of at least a regular part $(J)$ or a short-fat $\left(L^{T}\right)$ block. We call such a vector $\mathbf{x}$ a right annihilating vector of the pencil associated with the annihilating value $\lambda$. These are also a generalized eigenvector and value, respectively, if they are associated with the regular part, or if there is no short-fat part. If there is a short-fat part, then every complex number (including infinity) is an annihilating value, but only a finite number of these can be generalized eigenvalues as well. The eigenvalues, if any, will be exactly those values of $\lambda$ at which the matrix $A-\lambda B$ has a rank less than the overall maximum rank.

The work in this paper was motivated by the many roles matrix pencils play in control systems theory. We give one example below. Matrix pencils also play roles in the theory of transmission zeros and in the theory of differential algebraic equations.

Consider a time-invariant linear system

$$
\dot{\mathbf{x}}=F \mathbf{x}+G \mathbf{u} ; \mathbf{y}=H \mathbf{x}+D \mathbf{u} .
$$

A classical result from control theory is the Popov-Belevitch-Hautus (PBH) test (see, e.g., [11]), which states that the system (1) is controllable if and only if the matrix pencil

$$
P^{T}(\lambda)=[\lambda I-F \mid G]=[-F \mid G]-\lambda[-I \mid 0]
$$

has full rank for any complex value of $\lambda$. From a numerical point of view, one may say that if pencil (2) has a small singular value for some value of $\lambda$, then a small perturbation to the coefficients to (1) can yield an uncontrollable system [12].

We mention the main results from the perturbation theory of eigenvalues that we use in this paper. The most important result is the modified Bauer-Fike theorem, which gives bounds on the changes of the eigenvalues under perturbations in the underlying matrix.

Proposition 1 (modified Bauer-Fike theorem [6], [9]). We are given an $n \times n$ matrix $A$ with a complete set of eigenvalues $\lambda_{1}, \cdots, \lambda_{n}$ and corresponding left and right eigenvectors $\mathbf{w}_{1}, \cdots, \mathbf{w}_{n}, \mathbf{v}_{1}, \cdots, \mathbf{v}_{n}$. Let $V:=\left[\mathbf{v}_{1}, \cdots, \mathbf{v}_{n}\right]$ be the matrix of eigenvectors. Let $\Delta$ be another arbitrary $n \times n$ matrix, and let $\bar{\lambda}$ be any eigenvalue of $A+\Delta$. Then for at least one $\lambda_{i}, 1 \leq i \leq n$, the following bound holds:

$$
\left|\bar{\lambda}-\lambda_{i}\right| \leq K_{i}\|\Delta\|
$$

where

$$
K_{i} \equiv \min \left(\|V\| \cdot\left\|V^{-1}\right\|, \frac{n}{s_{i}}\right) \text { with } s_{i} \equiv \frac{\left|\mathbf{w}_{i}^{H} \mathbf{v}_{i}\right|}{\left\|\mathbf{w}_{i}\right\| \cdot\left\|\mathbf{v}_{i}\right\|}
$$

On (4) we remark that $\max _{i} s_{i}^{-1} \leq\|V\| \cdot\left\|V^{-1}\right\| \leq s_{1}^{-1}+\cdots+s_{n}^{-1} \leq n \cdot \max _{i} s_{i}^{-1}$ [18, pp. 88-89], so that these quantities are closely related. We use this definition for $K_{i}$ instead of just $\|V\| \cdot\left\|V^{-1}\right\|$ as in the original Bauer-Fike theorem because for some $i$ this may yield a somewhat tighter bound. The bounds will be noticeably tighter only for those $i$ for which $s_{i}$ is much larger than some other $s_{j}, j \neq i$, if there are any. 
We also use the following results regarding the changes to the eigenvectors under perturbations to a matrix $A$. We quote three different, but related, bounds in the following proposition and compare how they fare in the context of our matrix pencil problem.

Proposition 2 (Stewart [14], Boley (Appendix), Demmel [4], [5]). Let A be some arbitrary $n \times n$ matrix, which we assume for simplicity has distinct eigenvalues. Let $\mathbf{v}_{i}, i=1, \cdots, n$ be the eigenvectors of $A$, all of unit length. Let $A+\Delta$ be another arbitrary matrix, and let $\overline{\mathbf{v}}$ be some eigenvector of $A+\Delta$. Let $\theta_{i}$ be the angle between $\overline{\mathbf{v}}$ and $\mathbf{v}_{i}$, for $i=1, \cdots, n$. Finally, define $\operatorname{isep}_{A}\left(\lambda_{i}\right)[14]$ as $\left\|\left(R_{22}-\lambda_{i} I\right)^{-1}\right\|$, where $R_{22}$ is the trailing $(n-1) \times(n-1)$ block in the the Schur Decomposition of A:

$$
P^{H} A P=R=\left[\begin{array}{cc}
\lambda_{i} & R_{12} \\
0 & R_{22}
\end{array}\right] .
$$

Then if $\|\Delta\|$ is small enough to satisfy the condition given below for all $i$, then the tangent of at least one angle $\theta_{i}$ can be bounded by the corresponding expression. We have three closely related bounds:

(a) (Stewart [14]) If for all $i$

$$
\|\Delta\| \leq \frac{1}{4 \cdot \operatorname{isep}_{A}\left(\lambda_{i}\right) \cdot\left(1+\|A\| \cdot \operatorname{isep}_{A}\left(\lambda_{i}\right)\right)}
$$

then for at least one $i$

$$
\tan \theta_{i} \leq \gamma_{i}^{\mathbf{a}} \equiv \frac{\operatorname{isep}_{A}\left(\lambda_{i}\right)\|\Delta\|}{1-2 \cdot \operatorname{isep}_{A}\left(\lambda_{i}\right)\|\Delta\|} .
$$

(b) (see Appendix) If for all $i$

$$
\|\Delta\| \leq \frac{1}{\operatorname{isep}_{A}\left(\lambda_{i}\right) \cdot\left(1+K_{i}\right)}
$$

then for at least one $i$

$$
\tan \theta_{i} \leq \gamma_{i}^{\mathbf{b}} \equiv \frac{\operatorname{isep}_{A}\left(\lambda_{i}\right)\|\Delta\|}{1-\operatorname{isep}_{A}\left(\lambda_{i}\right)\|\Delta\|\left(1+K_{i}\right)} .
$$

Note that (5b) means that (6b) applies whenever the denominator is positive.

(c) (Demmel [4], [5]) If for all $i$

$$
\|\Delta\| \leq \frac{1}{4 \cdot \operatorname{isep}_{A}\left(\lambda_{i}\right) \cdot s_{i}^{-1}}
$$

then for at least one $i$

$$
\tan \theta_{i} \leq \gamma_{i}^{\mathbf{c}} \equiv \frac{4 \cdot \operatorname{isep}_{A}\left(\lambda_{i}\right) \cdot\|\Delta\|}{1-4 \cdot \operatorname{isep}_{A}\left(\lambda_{i}\right) \cdot\|\Delta\| \cdot \sqrt{s_{i}^{-2}-1}} .
$$

In each case above, $\overline{\mathbf{v}}$ can be scaled so that for some $i$

$$
\left\|\overline{\mathbf{v}}-\mathbf{v}_{i}\right\|=\sin \theta_{i} \leq \frac{\gamma_{i}^{\mathrm{x}}}{\sqrt{\left(\gamma_{i}^{\mathrm{x}}\right)^{2}+1}}
$$


where $\gamma_{i}^{\mathrm{x}}$ is defined by $(6 \mathrm{x}), \mathrm{x}=\mathrm{a}, \mathrm{b}, \mathrm{c}$, whenever these formulas apply.

Asymptotically as $\|\Delta\|$ goes to zero, all three bounds are the same, at least qualitatively, but we mention all three because each may yield the tighter bound for different values of $\|\Delta\|$. For example, it is evident that (6a) is tighter than (6b) when they both apply according to (5a) and (5b); but when (6a) does not apply, (6b) may still apply and hence be the tighter bound. Likewise, since the limit $(5 \mathrm{c})$ is the largest, the bound (6c) applies over the widest range for $\|\Delta\|$; it can, however, be less tight than (6a) and/or (6b) when they all apply. The numerical examples below will illustrate how one bound is best in some cases and another bound is best in other cases, but qualitatively they are all similar. As the anonymous reviewers pointed out, all these bounds can, and should, be further refined.

The rest of this paper is organized as follows. First we examine a method for computing whether or not a given pencil is deficient. Next we develop an upper bound for the distance to the nearest deficient pencil, and finally we develop a lower bound for this distance, using the eigensystem perturbation theory outlined above. We end with some numerical examples and conclusions. In the Appendix, we briefly sketch the derivation of the eigenvector bound $(6 \mathrm{~b})$.

2. Find pencil rank deficiency. In this section we address the problem of determining whether a given rectangular pencil is deficient or not. Specifically, given an $n \times p$ pencil $A-\lambda B$, with $n>p$, determine whether or not $A-\lambda B$ loses rank for any $\lambda$, including possibly $\lambda$ infinite. This is equivalent to asking whether or not the pencil has any short-fat K-blocks or regular part. If $B$ has full column rank, and the pencil never loses rank for any finite value of $\lambda$, then there are no short-fat blocks and no regular part.

Consider an $n \times p$ pencil $A-\lambda B$ with $n>p$. Choose arbitrary $n \times(n-p)$ matrices $C, D$. We can then examine the square $n \times n$ generalized eigenvalue problem

$$
[A, C] \mathbf{v}=\lambda[B, D] \mathbf{v} .
$$

We partition the vector $\mathbf{v}$ as $\mathbf{v}^{T} \equiv\left[\mathbf{x}^{T}, \mathbf{y}^{T}\right]$, where $\mathbf{x}$ is a $p$-vector, and $\mathbf{y}$ is an $(n-p)$-vector. It is then a simple matter to derive the following proposition.

Proposition 3. Given an $n \times p$ pencil $A-\lambda B$ with $n>p$, and given arbitrary full-rank $n \times(n-p)$ matrices $C, D$, the following are equivalent:

(a) $A-\lambda B$ is a deficient pencil.

(b) Equation (7) has an annihilating vector $\mathbf{v}_{0}$ whose last $n-p$ components $\mathbf{y}_{0}$ are zero. Call the corresponding annihilating value $\lambda_{0}$.

Furthermore, we have the following:

(c) If $B$ has full column rank, then all the annihilating vectors $\mathbf{v}_{0}$ and corresponding values $\lambda_{0}$ are exactly the generalized eigenpairs for the regular part of the pencil.

Proof. Both (a) and (b) are equivalent to the following statement:

(d) There is an $n$-vector $\mathbf{x}$ and scalar $\lambda_{0}$ which satisfies $A \mathbf{x}=\lambda_{0} B \mathbf{x}$, or else $B \mathbf{x}=0$. In the latter case, we say $\lambda_{0}=\infty$. If $B$ has full column rank, then there can be no short-fat blocks. Hence it follows that there is a regular part, and that the $\lambda_{0}$ 's are exactly the eigenvalues of that regular part. Otherwise, there is no regular part.

Based on this proposition, we have a simple procedure for computing the existence of a regular part or short-fat block in a pencil. Given a tall-thin $n \times p$ pencil, choose the $n \times(n-p)$ augmentation matrices $C, D$ to obtain the square eigenvalue problem 
(7). If there are any annihilating vectors of (7) whose last $n-p$ entries are zero, then there is a regular part or short-fat block; otherwise, there is not.

In the special case that $B=[I, 0]^{T}$, choose $D=[0, I]^{T}$ to turn (7) into an ordinary eigenproblem, and the annihilating vectors above into ordinary eigenvectors. If the last $n-p$ entries of any of those eigenvectors are zero, then the corresponding eigenvalues are exactly the eigenvalues of the regular part of the original pencil. Otherwise, there is no regular part.

However, this method gives no hint as to the sensitivity of the result to perturbations in the coefficients. Therefore, in the next sections, we develop some bounds that indicate whether a given pencil is "numerically close" to a deficient pencil.

3. Upper bounds. In this section, we examine the problem of computing an upper bound on the distance to a deficient pencil. Specifically, consider a nondeficient $n \times p$ pencil $A-\lambda B$. In this case, we know that $B$ has full rank. We would like to estimate the size of the perturbation $E$ to the matrix $A$ that is needed to obtain a deficient pencil $A+E-\lambda B$. This perturbed pencil will have a regular part, but no "short-fat" blocks. In this section we develop a simple upper bound for $\|E\|$.

In [7] and [12], it was shown that the smallest perturbation $E$ can be obtained by solving the minimization problem

$$
\min _{s} \sigma_{\min }(A-s B)
$$

where $\sigma_{\min }(M)$ denotes the smallest singular value of the matrix $M$, and $s$ varies over the entire complex plane. If we denote by $\sigma^{*}$ and $s^{*}$ the minimum in (8) and the value of $s$ achieving that minimum, respectively, then $\|E\|=\sigma^{*}$. In [3], we discussed an expensive descent method that would often converge to the minimum (8). In this section, we would like to address a much simpler scheme that can be used to obtain an upper bound, which often not only provides a good estimate for $\|E\|$, but also provides an estimate for that value of $s$ that yields the minimum in (8).

We start with the $n \times p$ pencil $A-\lambda B$. Choose some arbitrary full-rank $n \times(n-p)$ matrices $C, D$. And, in the case $B=[I, 0]^{T}$, choose $D=[0, I]^{T}$. Let

$$
\lambda_{i}, \mathbf{v}_{i} \equiv\left[\begin{array}{l}
\mathbf{x}_{i} \\
\mathbf{y}_{i}
\end{array}\right], i=1, \cdots, k
$$

be the generalized eigenvalues and vectors for (7), where $\mathbf{x}_{i}$ denotes the first $p$ components of $\mathbf{v}_{i}$. For each $i$, we have the equation $[A, C] \mathbf{v}_{i}=\lambda_{i}[B, D] \mathbf{v}_{i}$. We can rewrite this as $\left(\lambda_{i} B-A\right) \mathbf{x}_{i}=\left(C-\lambda_{i} D\right) \mathbf{y}_{i}$. We define the residual for each $i$ by $\mathbf{r}_{i}:=\left(A-\lambda_{i} B\right) \mathbf{x}_{i}$, and the perturbation $E_{i}$ to be

$$
E_{i}:=-\frac{\mathbf{r}_{i} \mathbf{x}_{i}^{T}}{\left\|\mathbf{x}_{i}\right\|^{2}} \equiv\left(\lambda_{i} B-A\right) \frac{\mathbf{x}_{i} \mathbf{x}_{i}^{T}}{\left\|\mathbf{x}_{i}\right\|^{2}}=\left(C-\lambda_{i} D\right) \frac{\mathbf{y}_{i}}{\left\|\mathbf{x}_{i}\right\|} \cdot \frac{\mathbf{x}_{i}^{T}}{\left\|\mathbf{x}_{i}\right\|}
$$

Then $A+E_{i}-\lambda B$ is a deficient pencil, losing rank exactly at $\lambda=\lambda_{i}$, for each $i$. Let $\sigma_{i}, \mathbf{u}_{i}, \mathbf{w}_{i}$ be, respectively, the smallest singular value and the corresponding left and right singular vectors of $A-\lambda_{i} B$, for each $i$. Then $E_{i}^{\prime}:=-\sigma_{i} \mathbf{u}_{i} \mathbf{w}_{i}^{T}$ is another smaller perturbation yielding a deficient pencil.

By taking norms of (10), we obtain a bound for these perturbations: $\left\|E_{i}^{\prime}\right\| \leq$ $\left\|E_{i}\right\| \leq\left\|\left(C-\lambda_{i} D\right) \mathbf{y}_{i}\right\| /\left\|\mathbf{x}_{i}\right\|$. If $E$ denotes that perturbation with smallest norm yielding a deficient pencil, then $E$ satisfies

$$
\begin{aligned}
\|E\| & \equiv \sigma^{*} \leq \beta_{2} \equiv \min _{i}\left\|E_{i}^{\prime}\right\| \equiv \min _{i} \sigma_{\min }\left(A-\lambda_{i} B\right) \\
& \leq \beta_{1} \equiv \min _{i}\left\|E_{i}\right\| \equiv \min _{i} \frac{\left\|\left(C-\lambda_{i} D\right) \mathbf{y}_{i}\right\|}{\left\|\mathbf{x}_{i}\right\|}
\end{aligned}
$$


Regarding the two bounds $\beta_{1}, \beta_{2}$, we remark that $\beta_{1}$ can be computed directly from the solution to the eigenproblem (7), whereas $\beta_{2}$ requires computing the singular value decomposition (SVD) at some extra expense. We can summarize the result in the following proposition.

Proposition 4. Let $A-\lambda B$ be an $n \times p$ pencil, with $n>p$. Let $C, D$ be two arbitrary full-rank $n \times(n-p)$ matrices. Then the smallest perturbation $E$ such that $A+E-\lambda B$ is a deficient pencil satisfies the bound (11), where $\lambda_{i}, \mathbf{v}_{i}, i=1, \cdots, k$ are the eigenpairs of the generalized eigenproblem (7), and $\mathbf{y}_{i}$ are defined by (9).

Proof: This follows from the above discussion. All we must note is that from Proposition 3, if the pencil $A-\lambda B$ is already deficient, then $E=0$ automatically satisfies (11). In fact, if $B$ has full column rank, one of the $\mathbf{y}_{i}$ should be zero by Proposition 3, so the bound will be hard.

One question is how to choose $C, D$. One goal is to make the augmented square eigenproblem as well conditioned as possible. So far, the only requirement we have stated is that $C, D$ have full column rank. To keep the condition number as low as possible, it is best to choose $C, D$ to each have orthonormal columns. Two possible choices are (a) orthonormal basis of a random space, and (b) orthonormal basis of the space orthogonal to the columns of $A$ and $B$. This last choice has the effect of limiting the increase to the condition numbers of $[A, C]$ and $[B, D]$ with respect to inversion, and hence is a heuristic attempt to obtain a reasonably low condition number with respect to the eigenproblem. In any case, the algorithms are intended to provide a posteriori estimates for a given pencil, and in that context it is easy to check that the condition number of the resulting eigenproblem is reasonably small. Most of the numerical examples below were carried out with choice (b). We note that in the special case $B=[I, 0]^{T}$, we choose $D=[0, I]^{T}$ to turn (7) into an ordinary eigenproblem.

4. Lower bounds. In this section, we show how to extend the results of the previous section for the special case of pencils $A-\lambda B$ such that $B=[I, 0]^{T}$, to obtain some lower bounds and to obtain a disk in the complex plane in which the value $s$ achieving the minimum in (8) must be located. The first lower bound is based just on the Bauer-Fike theorem whereas the other lower bounds are based on the eigenvector perturbation theorem (Proposition 2). It will be seen that the first lower bound is not as tight as the others, but it is much simpler to derive and much cheaper to compute, since it does not require the "isep" function.

Let $s^{*}$ be the complex value achieving the minimum in (8), and let $\sigma^{*}$ be the smallest singular value of $A-s^{*} B$. Augment $A-s B$ as before with extra columns $C$ and $D=[0, I]^{T}$, obtaining the square matrix $[A, C]$, so that $(7)$ becomes the ordinary eigenproblem for the matrix $[A, C]$. Then the smallest singular value $\tau$ of $[A, C]-s^{*} I$ satisfies $\tau \leq \sigma^{*}$, since augmenting with extra columns can only reduce the smallest singular value [9]. So $s^{*}$ is an exact eigenvalue of $[A, C]+\tau \Delta$, for some matrix $\Delta$ such that $\|\Delta\|=1$. Denote the eigenvalues of $[A, C]$ by $\lambda_{1}, \cdots, \lambda_{n}$. Then, for at least one such eigenvalue, the modified Bauer-Fike theorem implies that $\left|\lambda_{i}-s^{*}\right| \leq \tau K_{i}$, where $K_{i}$ is defined in (4).

Next, let $\alpha$ be the smallest singular value of $A-\lambda_{i} B$. Then

$$
\left|\alpha-\sigma^{*}\right| \leq\left\|\left(A-\lambda_{i} B\right)-\left(A-s^{*} B\right)\right\| \leq\left|\lambda_{i}-s^{*}\right| \leq \tau K_{i} \leq \sigma^{*} K_{i} .
$$

From this formula, we can draw two conclusions. One is that $\alpha \leq \sigma^{*}\left(K_{i}+1\right)$, yielding 
the lower bound on $\sigma^{*}$ :

$$
\sigma^{*} \geq \frac{\alpha}{K_{i}+1} \geq \frac{\beta_{2}}{K_{i}+1}
$$

where $\beta_{2}$ is the upper bound $\beta_{2}$ defined in (11).

The other conclusion from (12) is

$$
\left|\lambda_{i}-s^{*}\right| \leq \sigma^{*} K_{i} \leq \beta_{2} K_{i} .
$$

We can summarize this in the following proposition.

Proposition 5. Given a pencil $A-s B$, where $B=[I, 0]^{T}$, and an arbitrary (full-rank) augmentation of this pencil to a square matrix $[A, C]$ as in $(7)$, then

(a) The value of $s$ that achieves the minimum in $\sigma^{*} \equiv \min _{s} \sigma_{\min }(A-s B)$ is located within a disk in the complex plane whose center is on an eigenvalue $\lambda_{i}$ of $Q$ and whose radius is bounded by (14), for some $i$.

(b) A lower bound on $\sigma^{*}$ is provided by (13).

By using the eigenvector bounds in Proposition 2, we can derive some tighter lower bounds on $\sigma^{*}$. We base our development on $(5 \mathrm{a}) /(6 \mathrm{a})$, but by analogy the exact same development goes through with $(5 \mathrm{~b}) /(6 \mathrm{~b})$ or with $(5 \mathrm{c}) /(6 \mathrm{c})$. Given a pencil $A-s B$ with $B=[I, 0]^{T}$, the eigenvectors (9) of the augmented matrix $[A, C]$ are defined. If $A+E-s B$ is a deficient pencil, then the matrix $[A+E, C]$ must have at least one eigenvector of the form $\overline{\mathbf{v}}=\left[\overline{\mathbf{x}}^{T}, 0\right]^{T}$, where $\overline{\mathbf{v}}$ is partitioned as in (9). That is, the square matrix $[A, C]$ must be perturbed to a matrix which has an eigenvector $\overline{\mathbf{v}}$ whose $\mathbf{y}$ part is zero. But then the bound $(5 \mathrm{a}) /(6 \mathrm{a})$ directly yields a lower bound on the norm of the perturbation to $[A, C]$ so that an eigenvector of the resulting matrix has the indicated form. The resulting lower bound is

$$
\sigma^{*} \geq \delta^{\mathbf{a}} \equiv \min _{i} \min \left\{\begin{array}{ll}
\text { (i) } & \frac{\left[\operatorname{isep}_{A}^{-1}\left(\lambda_{i}\right)\right]^{2}}{4\left(\operatorname{isep}_{A}^{-1}\left(\lambda_{i}\right)+\|A\|\right)}, \\
\text { (ii) } \frac{\eta_{i}}{\operatorname{isep}_{[A, C]}\left(\lambda_{i}\right)\left(1+2 \eta_{i}\right)}
\end{array}\right\},
$$

where

$$
\eta_{i} \equiv \frac{y_{i}}{\sqrt{1-y_{i}^{2}}} .
$$

Bounds (i),(ii) come from (5a),(6a), respectively. Alternatively, we can use part (b) of Proposition 2 (this always satisfies the limit (5b)):

$$
\sigma^{*} \geq \delta^{\mathbf{b}}=\min _{i} \frac{\eta_{i}}{\operatorname{isep}_{[A, C]}\left(\lambda_{i}\right)\left(1+\eta_{i}\left(1+K_{i}\right)\right)}
$$

or part (c) of Proposition 2:

$$
\sigma^{*} \geq \delta^{\mathbf{c}}=\min _{i} \min \left\{\begin{array}{l}
\text { (i) } \frac{1}{4 \cdot \operatorname{isep}_{[A, C]}\left(\lambda_{i}\right) \cdot s_{i}^{-1}}, \\
\text { (ii) } \frac{\eta_{i}}{4 \cdot \operatorname{isep}_{[A, C]}\left(\lambda_{i}\right)\left(1+\eta_{i} \sqrt{s_{i}^{-2}-1}\right)}
\end{array}\right\} .
$$

We note that the backward stability of these methods depends on the backward stability of the method used to obtain the eigendecompositions. If the eigenvalue 
method is backward stable, then these bounds will be exact for a pencil numerically close to the original pencil, and the residual from the eigenvalue method will indicate how far from that original pencil we have strayed. But in the cases we have tried, the size of this residual was never more than $1 \mathrm{E}-11$, much less than the computed bounds themselves.

5. Numerical examples. We illustrate the bounds with the examples taken from [3]. Each example represents a time-invariant linear system of the general form $\dot{\mathbf{x}}=F \mathbf{x}+G \mathbf{u}$, from which we form the pencil (2). Example 1 is defined by

$$
F=\left[\begin{array}{cc}
0 & 1 \\
-1 & 0
\end{array}\right] \text { and } G=\left[\begin{array}{l}
1 \\
0
\end{array}\right]
$$

In Example 2, we start with a single-input system already in staircase form [13], [15], with $G=[1,0, \cdots, 0]^{T}$, and

$$
F=\left[\begin{array}{rrrrrrr}
-1 & -1 & -1 & -1 & -1 & -1 & 7 \\
1 & -1 & -1 & -1 & -1 & -1 & 6 \\
0 & 1 & -1 & -1 & -1 & -1 & 5 \\
0 & 0 & 1 & -1 & -1 & -1 & 4 \\
0 & 0 & 0 & 1 & -1 & -1 & 3 \\
0 & 0 & 0 & 0 & 1 & -1 & 2 \\
0 & 0 & 0 & 0 & 0 & 1 & 1
\end{array}\right]
$$

Example 3 is one with a particularly ill-conditioned eigenvalue problem. The system is defined by

$$
F=\left[\begin{array}{rrr}
-149 & 537 & -27 \\
-50 & 180 & -9 \\
-154 & 546 & -25
\end{array}\right] \text { and } G=\left[\begin{array}{l}
1 \\
1 \\
1
\end{array}\right]
$$

The poles (eigenvalues) for this system are 1, 2, and 3 .

The staircase algorithm [13], [1] applied to a single input system (i.e. $G$ has only one column) transforms $F$ into an upper Hessenberg form, with $G$ a multiple of $\mathbf{e}_{1}$. The pencil (2) has a regular part if and only if a subdiagonal element of the Hessenberg form is zero, so an obvious upper bound is simply the magnitude of the smallest subdiagonal element. This is the second column of Table 1. In the third column are shown upper bounds obtained by the expensive experimental descent method described in [3]. In the last column of Table 1 are lower bounds from the theory in [2], which was based on using the product of the subdiagonal elements.

We report in Table 2 the upper bounds computed using the formula (11). It is seen that the bound $\beta_{2}$ is always tighter than $\beta_{1}$ and is in fact fairly close to the "optimal" upper bound reported in Table 1. In Table 3 we report the various lower bounds. For reference, we copied the tightest lower bound found for each example to Table 2 to show the spread between the upper and lower bounds. It is seen that the best lower bound is obtained from formula (15a) (ii) when it applies; otherwise, the tightest bound is obtained from (15b). We note that upper bound 1 and lower bound 0 take only $O(n+p)^{3}$ work to obtain, so the computation is relatively fast. Upper bound 2 requires computing the smallest singular value at some extra expense, but we do not address here possible ways to speed this up. The other lower bounds would also be fast to obtain, except for the computation of the rather expensive "isep" 
function. Note that the upper bounds from [3] in Table 1 are tighter, but are much more expensive to obtain.

In Table 4, we give the eigenvalue $\lambda_{i}$ of the augmented matrix at which the upper bounds were taken, together with the radius (14) about this value within which the minimum in (8) is located.

TABLE 1.

Bounds from older methods.

\begin{tabular}{cccc}
\hline $\begin{array}{c}\text { Example } \\
\#\end{array}$ & $\begin{array}{c}\text { Upper bound } \\
\text { staircase }[1]\end{array}$ & $\begin{array}{c}\text { Upper bound } \\
\text { from [3] }\end{array}$ & $\begin{array}{c}\text { Lower bound } \\
\text { from }[2]\end{array}$ \\
\hline 1 & 1.0 & $6.6144 \mathrm{E}-01$ & $1.2500 \mathrm{E}-01$ \\
2 & 1.0 & $6.7690 \mathrm{E}-04$ & $4.3654 \mathrm{E}-08$ \\
3 & $1.1610 \mathrm{E}-02$ & $4.3715 \mathrm{E}-03$ & $8.5774 \mathrm{E}-07$ \\
\hline
\end{tabular}

TABLE 2 .

Upper bounds from formula (11).

\begin{tabular}{cccc}
\hline $\begin{array}{c}\text { Example } \\
\#\end{array}$ & $\begin{array}{c}\text { Upper } \\
\text { bound } 1 \\
\beta_{1}\end{array}$ & $\begin{array}{c}\text { Upper } \\
\text { bound } 2 \\
\beta_{2}\end{array}$ & $\begin{array}{c}\text { Best lower } \\
\text { bound from } \\
\text { Table } 3\end{array}$ \\
\hline 1 & $7.2561 \mathrm{E}-01$ & $7.0545 \mathrm{E}-01$ & $3.7272 \mathrm{E}-01(15 \mathrm{~b})$ \\
2 & $8.8790 \mathrm{E}-04$ & $7.3074 \mathrm{E}-04$ & $6.5105 \mathrm{E}-04(15 \mathrm{a})$ \\
3 & $1.1507 \mathrm{E}-02$ & $4.6607 \mathrm{E}-03$ & $1.0313 \mathrm{E}-03(15 \mathrm{~b})$ \\
\hline
\end{tabular}

TABLE 3.

Lower bounds from methods in this paper.

\begin{tabular}{ccccc}
\multicolumn{6}{c}{ (Bounds from equation } & (15) come from (ii) except those marked "*.") \\
\hline $\begin{array}{c}\text { Example } \\
\#\end{array}$ & $\begin{array}{c}\text { Lower } \\
\text { bound 0 } \\
(13)\end{array}$ & $\begin{array}{c}\text { Lower } \\
\text { bound a } \\
(15 \mathrm{a})\end{array}$ & $\begin{array}{c}\text { Lower } \\
\text { bound b } \\
(15 \mathrm{~b})\end{array}$ & $\begin{array}{c}\text { Lower } \\
\text { bound c } \\
(15 \mathrm{c})\end{array}$ \\
\hline 1 & $3.1480 \mathrm{E}-01$ & $* 1.9409 \mathrm{E}-01$ & $3.7272 \mathrm{E}-01$ & $1.7264 \mathrm{E}-01$ \\
2 & $7.2095 \mathrm{E}-05$ & $6.5105 \mathrm{E}-04$ & $6.4726 \mathrm{E}-04$ & $1.6279 \mathrm{E}-04$ \\
3 & $8.6385 \mathrm{E}-04$ & $* 1.7989 \mathrm{E}-05$ & $1.0313 \mathrm{E}-03$ & $2.6339 \mathrm{E}-04$ \\
\hline
\end{tabular}

TABLE 4.

Values of $\lambda$ at which upper bounds in Table 2 were obtained.

\begin{tabular}{ccc}
\hline $\begin{array}{c}\text { Example } \\
\#\end{array}$ & $\begin{array}{c}\lambda_{i} \text { achieving min in } \\
\min _{i} \sigma_{\min }\left(A-\lambda_{i} B\right) \equiv \beta_{2} \\
(11)\end{array}$ & $\begin{array}{c}\text { Radius about } \\
\text { given } \lambda_{i} \\
(14)\end{array}$ \\
\hline 1 & $-1.6899 \mathrm{E}-01+1.1509 \mathrm{E}+00 i$ & $8.7545 \mathrm{E}-01$ \\
2 & $+1.9998 \mathrm{E}+00+6.5937 \mathrm{E}-16 i$ & $6.6758 \mathrm{E}-03$ \\
3 & $+2.4534 \mathrm{E}+00+0.0000 \mathrm{E}+00 i$ & $2.0485 \mathrm{E}-02$ \\
\hline
\end{tabular}

When the staircase algorithm is applied to Example 3, we obtain (items in parenthesis are close to the machine epsilon) the following:
$F$ new $=$

$\begin{array}{rcr}2.8300 \mathrm{E}+02 & 6.9026 \mathrm{E}+02 & -1.3400 \mathrm{E}+02 \\ -1.1458 \mathrm{E}+02 & -2.7946 \mathrm{E}+02 & 5.4837 \mathrm{E}+01 \\ (-1.6584 \mathrm{E}-15) & -1.1610 \mathrm{E}-02 & 2.4570 \mathrm{E}+00\end{array}$
Gnew $=$
$-1.7321 \mathrm{E}+00$
$(-2.5339 \mathrm{E}-16)$
(4.2062E-16) 
In Table 5, we illustrate the effect of using a different way to augment the matrix rather than an orthonormal basis to the space orthogonal to the column space of $A$. These numbers were obtained with Example 2. The first two lines were obtained using an orthonormal basis for two different random spaces. The third line was obtained by using random columns, not orthonormal, but with elements uniformly distributed in the interval $[-1,1]$. The fourth line was obtained by adding a random perturbation to $A$ of norm $1 \mathrm{E}-5$ and then following the original prescription used for Tables 2 and 3 . The fifth line was copied from Table 2 for comparison. Generally, the bounds from Table 2 are at least as tight, except for the SVD-based upper bound $\beta_{2}$ (11), for which using a random set of orthonormal columns was better.

TABLE 5 .

Bounds on Example 2using different random schemes.

\begin{tabular}{ccccc}
\hline $\begin{array}{c}\text { Example } \\
\#\end{array}$ & $\begin{array}{c}\text { Upper } \\
\text { bound 1 } \\
\beta_{1}(11)\end{array}$ & $\begin{array}{c}\text { Upper } \\
\text { bound 2 } \\
\beta_{2}(11)\end{array}$ & $\begin{array}{c}\text { Lower } \\
\text { bound 0 } \\
(13)\end{array}$ & $\begin{array}{c}\text { Best lower } \\
\text { bound, all } \\
\text { from (15a) }\end{array}$ \\
\hline Rand 1 & $7.6641 \mathrm{E}-04$ & $6.8228 \mathrm{E}-04$ & $6.5806 \mathrm{E}-05$ & $6.4203 \mathrm{E}-04$ \\
Rand 2 & $7.6697 \mathrm{E}-04$ & $6.8038 \mathrm{E}-04$ & $6.5224 \mathrm{E}-05$ & $6.3977 \mathrm{E}-04$ \\
Non-ortho & $8.9135 \mathrm{E}-04$ & $6.8256 \mathrm{E}-04$ & $6.3614 \mathrm{E}-05$ & $6.0989 \mathrm{E}-04$ \\
Perturbed & $8.8983 \mathrm{E}-04$ & $7.3232 \mathrm{E}-04$ & $7.2426 \mathrm{E}-05$ & $6.5245 \mathrm{E}-04$ \\
Table 2 & $8.8790 \mathrm{E}-04$ & $7.3074 \mathrm{E}-04$ & $7.2095 \mathrm{E}-05$ & $6.5105 \mathrm{E}-04$ \\
\hline
\end{tabular}

6. Conclusions. We have given a scheme for estimating the distance from a given pencil to the nearest pencil of different Kronecker structure. In the context of dynamical systems, this yields estimates of the distance to the nearest uncontrollable system. Unlike the staircase-type algorithms, the scheme presented here does not depend on the recursive computation of the singular values of small matrix subblocks, so it is less sensitive to the particular choice of zero tolerance. Though there is no a priori guarantee that the bounds obtained using the methods from this paper will be good, the spread between the upper and lower bounds will automatically give a measure of the quality of the bounds themselves. Furthermore, since we also have localized the location of the minimum in (8) to within certain small disks, we also have good values with which to start an iterative procedure to refine the estimate of the location of this minimum (for example, using the experimental descent method proposed in [3], for which a good starting value is critical for successful convergence).

Appendix. We briefly sketch the derivation of the bound (6b). Let $A$ be a matrix with a simple eigenvalue $\lambda$ and associated eigenvector $\mathbf{v}$, with $\|\mathbf{v}\|=1$. We can then find a unitary matrix $P$ whose first column is $\mathbf{v}$ such that

$$
P^{H} A P=R=\left[\begin{array}{ll}
\lambda & R_{12} \\
0 & R_{22}
\end{array}\right],
$$

where $R_{22}$ is an $(n-1) \times(n-1)$ matrix, none of whose eigenvalues equals $\lambda$. The eigenvector of $R$ corresponding to $\lambda$ is $\mathbf{e}_{1} \equiv[1,0, \cdots, 0]^{T}$. We examine how this eigenvector changes under perturbations $E$ to $R$. Let $E$ be a (small) perturbation matrix and let $\bar{\lambda}$ be any eigenvalue of $R+E$, with corresponding eigenvector $\mathbf{f} \equiv\left[1, \mathbf{x}^{T}\right]^{T}$, partitioned conformally with (A1), and which is scaled to have first component equal 
to 1 . If $\theta$ is the angle between $\mathbf{e}_{1}$ and $\mathbf{f}$, then $\|\mathbf{x}\| \equiv \tan \theta$. We then have the relation

$$
0=(R+E-\bar{\lambda} I) \mathbf{f}=\left[\begin{array}{cc}
\lambda+e_{11}-\bar{\lambda} & R_{12}+E_{12} \\
E_{21} & R_{22}+E_{22}-\bar{\lambda} I
\end{array}\right]\left[\begin{array}{l}
1 \\
\mathbf{x}
\end{array}\right]
$$

where

$$
E \equiv\left[\begin{array}{ll}
e_{11} & E_{12} \\
E_{21} & E_{22}
\end{array}\right]
$$

is partitioned as in (A1). Define the $(n-1) \times(n-1)$ matrices $M \equiv R_{22}-\lambda I$, and $-N \equiv E_{22}+(\lambda-\bar{\lambda}) I$ and note that $M$ is nonsingular. If $\left\|M^{-1} N\right\|<1$, then $(M-N)^{-1}$ exists and can be bounded by $\left\|(M-N)^{-1}\right\| \leq\left\|M^{-1}\right\| /\left(1-\left\|M^{-1} N\right\|\right)$ [9]. Then the last $n-1$ equations of (A2) can be written as just $(M-N) \mathbf{x}=-E_{21}$, yielding the bound

$$
\|\mathbf{x}\| \leq \frac{\left\|M^{-1}\right\| \cdot\left\|E_{21}\right\|}{\operatorname{pos}\left(1-\left\|M^{-1} N\right\|\right)} \leq \frac{\left\|M^{-1}\right\| \cdot\|E\|}{\operatorname{pos}\left(1-\left\|M^{-1}\right\|(\|E\|+|\lambda-\bar{\lambda}|)\right)}
$$

where "pos" is a function defined by $\operatorname{pos}(r) \equiv r$ for $r>0$, and $\operatorname{pos}(r) \equiv 0$ for $r \leq 0$. The "pos" function simply expresses the fact that (A3) always holds, but only vacuously if the denominator is not positive. For example, this would occur if $\mathbf{f}$ is orthogonal to $\mathbf{e}_{1}$. We summarize the above in the following lemma.

LEMma A1. Let the upper triangular matrix $R$ be partitioned as in (A1), $\lambda, \mathbf{e}_{1}$ be a simple eigenpair for $R, M \equiv R_{22}-\lambda I, E$ be some arbitrary matrix, and $\bar{\lambda}$, $\mathbf{f}$ be any eigenpair for $R+E$. Then $\mathbf{f}$ can be scaled so that the difference $\mathbf{e}_{1}-\mathbf{f}$ satisfies $\left\|\mathbf{e}_{1}-\mathbf{f}\right\|=\sin \theta$, where $\tan \theta \equiv\|\mathbf{x}\|$ satisfies the bound (A3).

We note that if one expands (A3) in a power series in $\|E\|$, the first-order term will be identical to the first-order bound in [16]. Since $R$ and $A$ are related by a unitary transformation, this lemma leads directly to a corresponding bound for the change in the eigenvectors for an arbitrary matrix $A$. We can apply the modified Bauer-Fike theorem directly to the lemma to obtain the following proposition. We define the inverse "separation" function (following [14]) to be isep $A(\lambda) \equiv\left\|\left(R_{22}-\lambda I\right)^{-1}\right\|$.

Proposition A1. Let $A$ be some arbitrary $n \times n$ matrix with all distinct eigenvalues, and denote by $V$ the matrix of eigenvectors of $A$. Let $A+\Delta$ be another arbitrary matrix, and let $\bar{\lambda}, \overline{\mathbf{v}}$ be any eigenpair for $A+\Delta$. Let $\theta_{i}$ denote the angle between $\overline{\mathbf{v}}$ and the $i$-th unit eigenvector $\mathbf{v}_{i}$ of $A, i=1, \cdots, n$. Then for some $i$ we have the bound

$$
\tan \theta_{i} \leq \gamma_{i}^{\mathbf{b}} \equiv \frac{\operatorname{isep}_{A}\left(\lambda_{i}\right)\|\Delta\|}{\operatorname{pos}\left(1-\operatorname{isep}_{A}\left(\lambda_{i}\right)\|\Delta\|\left(1+K_{i}\right)\right)} .
$$

Furthermore, $\overline{\mathbf{v}}$ can be scaled so that we have the bound on the distance to some eigenvector $\mathbf{v}_{i}$ of $A$, for some $i$ :

$$
\left\|\mathbf{v}_{i}-\overline{\mathbf{v}}\right\|=\sin \theta_{i} \leq \frac{\gamma_{i}^{\mathbf{b}}}{\sqrt{1+\left(\gamma_{i}^{\mathbf{b}}\right)^{2}}}
$$

Note that this gives a nonvacuous bound as long as $\|\Delta\|$ is small enough to make the denominator in (A4) positive. 


\section{REFERENCES}

[1] D. Boley, A. Emami-Naeini, G. F. Franklin, A New Algorithm for Canonical Decomposition of Linear Systems, Proc. of 19th IEEE Conference on Decision and Control, Albuquerque, NM (1980), pp. 199-200.

[2] D. Boley, A perturbation result for linear control problems, SIAM J. Algebraic Discrete Methods, 6 (1985), pp. 66-72.

[3] — Computing the rank-deficiency of rectangular matrix pencils, Systems Control Lett., 9 (1987), pp. 207-214.

[4] J. Demmel, Computing stable eigendecompositions of matrices, Linear Algebra Appl., 79 (1986), pp. 163-193.

[5] _ Computing stable eigendecompositions of matrix pencils, Linear Algebra Appl., 88/89 (1987), pp. 139-186.

[6] - On condition numbers and the distance to the nearest ill-posed problem, Numer. Math., 51 (1987), pp. 251-289.

[7] R. Eising, Between Controllable and Uncontrollable, Systems Control Lett., 4 (1984), pp. 263-264.

[8] F. R. Gantmakher, Theory of Matrices, Vols 1 and 2, Celsea, New York, 1959.

[9] G. H. Golub, C. Van Loan, Matrix Computations, Johns Hopkins, Baltimore, MD, 1983.

[10] B. KÅGTROM, RGSVD - An algorithm for computing the Kronecker structure and reducing subspaces of singular $A-\lambda B$ pencils, SIAM J. Sci. Statist. Comput., 7 (1986), pp. 185-211.

[11] T. Kailath, Linear Systems; Prentice Hall, Englewood Cliffs, NJ, 1980.

[12] G. Miminis, Numerical algorithms for controllability and eigenvalue computation, Masters Thesis, School of Computer Science, McGill University, Montréal, Québec, Canada, May 1981.

[13] C. C. PAIGE, Properties of numerical algorithms related to controllability, IEEE Trans. Automat. Control, AC-26, 1 (1981), pp. 130-138.

[14] G. W. Stewart, Error and perturbation bounds associated with certain eigenvalue problems, SIAM Rev., 15 (1973), pp. 727-764.

[15] P. VAN DOOREn, The computation of Kronecker's canonical form of a singular pencil, Linear Algebra Appl., 27 (1979), pp. 103-141.

[16] J. VARAH, Invariant subspace perturbations for a nonnormal matrix, IFIP 71 Proceedings, North-Holland, Amsterdam, 1971.

[17] W. C. Waterhouse, The codimension of singular matrix pairs, Linear Algebra Appl., 57 (1984), pp. 227-245.

[18] J. H. Wilkinson, The Algebraic Eigenvalue Problem; Clarendon Press, Oxford, 1965. 